CUBO A Mathematical Journal

Vol.19, No 02, (49-71). June 2017

\title{
On topological symplectic dynamical systems
}

\author{
S. Tchuiaga $^{1}$, M. Koivogui ${ }^{2}$, F. Balibuno ${ }^{3}$ and V. Mbazumutima ${ }^{3}$ \\ ${ }^{1}$ Department of Mathematics \\ The University of Buea, South West Region, Cameroon. \\ tchuiagas@gmail.com \\ ${ }^{2}$ Ecole Supérieure Africaine des Technologies de l'Information et de Communication, \\ Côte d'Ivoire. \\ moussa.koivogui@esatic.ci \\ ${ }^{3}$ Institut de Mathématiques et des Sciences Physiques \\ Bénin. \\ balibuno.lugando@imsp-uac.org, mbazumutima.vianney@aims-cameroon.org
}

\begin{abstract}
This paper contributes to the study of topological symplectic dynamical systems, and hence to the extension of smooth symplectic dynamical systems. Using the positivity result of symplectic displacement energy [4, we prove that any generator of a strong symplectic isotopy uniquely determine the latter. This yields a symplectic analogue of a result proved by $\mathrm{Oh}$ [12, and the converse of the main theorem found in [6]. Also, tools for defining and for studying the topological symplectic dynamical systems are provided: We construct a right-invariant metric on the group of strong symplectic homeomorphisms whose restriction to the group of all Hamiltonian homeomorphism is equivalent to Oh's metric [12, define the topological analogues of the usual symplectic displacement energy for non-empty open sets, and we prove that the latter is positive. Several open conjectures are elaborated.
\end{abstract}




\section{RESUMEN}

Este artículo contribuye al estudio de los sistemas dinámicos simplécticos topológicos, y por tanto a la extensión de los sistemas dinámicos simplécticos suaves. Usando el resultado de la positividad de la energía de desplazamiento simpléctico 4, demostramos que cualquier generador de una isotopía simpléctica determina esta última. Esto entrega un análogo simpléctico de un resultado demostrado por Oh [12, y el inverso del teorema principal encontrado en [6]. También entregamos herramientas para definir y estudiar los sistemas dinámicos simplécticos topológicos: construimos una métrica invariante por derecha en el grupo de homeomorfismos fuertemente simplécticos cuya restricción al grupo de homeomorfismos Hamiltonianos es equivalente a la métrica de $\mathrm{Oh}$ [12, definimos los análogos topológicos de la energía de desplazamiento simpléctico usual para conjuntos no-vacíos, y demostramos que esta última es positiva. Planteamos varios problemas abiertos.

Keywords and Phrases: Isotopies, Diffeomorphisms, Homeomorphisms, Displacement energy, Hofer-like norms, Mass flow, Riemannian metric, Lefschetz type manifolds, Flux geometry.

2010 AMS Mathematics Subject Classification: 53D05, 53D35, 57R52, 53C21. 


\section{Introduction}

Gromov [10] showed that on a symplectic manifold, the $\mathrm{C}^{0}$-closure of the group of symplectic diffeomorphisms in the group of diffeomorphisms is either the group of symplectic diffeomorphisms itself, or the group of volume preserving diffeomorphisms. Eliashberg [9] proved that the symplectic nature of a sequence of symplectic diffeomorphisms survives topological limits. This result is known as the "celebrated rigidity" result of Eliashberg, which motivated various remarkable studies of continuum phenomena in the field of symplectic geometry. Especially, based on this rigidity result, Oh-Müller [13] defined the group of symplectic homeomorphisms as the $\mathrm{C}^{0}$-closure of the group of symplectomorphisms in the group of homeomorphisms. They also defined both versions of $\mathrm{C}^{0}$-Hamiltonian topologies on the space of Hamiltonians paths and used them to define the group of Hamiltonian homeomorphisms. This group is at the center of the study of topological Hamiltonian dynamical systems (see Viterbo [19, Buhovsky and Seyfaddini [8, 12]). More recently, motivated again by the celebrated rigidity result of Eliashberg, Banyaga [2, 3] defined two contexts of symplectic topologies on the space of symplectic isotopies that generalize the $\mathrm{C}^{0}$-Hamiltonian topologies. These topology had been used to define a new class of symplectic homeomorphisms named the "group strong symplectic homeomorphisms" (see Banyaga [3]), which had been studied in Banyaga-Tchuiaga [6, 5, 14]. This group could be the right topological analogue of the identity component in the group of symplectomorphisms. However, for that to be possible, we have to define, and then study what will be the equivalents (or analogues) of some well known smooth symplectic objects in the world (or context) of strong symplectic homeomorphisms.

Therefore, one purpose of this paper is to point out further studies of generators for strong symplectic isotopies [6], and then use them to construct a framework in which the flux homomorphism and the Hofer-like geometry can be extended to some category of continuous maps (see [18]).

We organize the present paper as follows: In Sections 2 and Section 3, we recall some fundamental tools needed in the definition of strong symplectic homeomorphisms: The description of symplectic isotopies that was introduced in [5], and the displacement energy. Section 4 deals with the definitions of the $\mathrm{C}^{0}$-compact open topology, the origin of strong symplectic homeomorphisms, and the definition of strong symplectic isotopies with their generators. These tools are used to show a bijective correspondence between the group of strong symplectic isotopies and the group of their generators (Theorem 4.4). The Hamiltonian version of this result is well known. This section also includes Lemma 4.6 which shows that any strong symplectic isotopy which is a 1-parameter group decomposes as composition of a smooth harmonic flow and a continuous Hamiltonian flow in the sense of Oh-Müller.

In Section 5, we use the results of Section 4 to introduce a topological version of Hofer-like geometry: We construct a topological counterpart of the Hofer-like metric for strong symplectic homeomorphisms, and we prove that its restriction to the group of Hamiltonian homeomorphisms is equivalent to Oh's metric [12]. Therefore, the definition of a topological analogue of the usual symplectic displacement for non-empty sets is given, and we prove that it is positive. Finally, in 
Section 6, we elaborate some conjectures and some examples are given.

\section{Preliminaries}

Let $M$ be an $2 n$-dimensional manifold of class $C^{\infty}$. A differential 2 -form $\omega$ on $M$ is called a symplectic form if it is closed and non-degenerate. The nondegeneracy of $\omega$, implies that $\omega^{n}$ is a volume form on $M$. A symplectic manifold is an even dimensional smooth manifold $M$ that admits a symplectic form $\omega$. From now on, we assume that $M$ is an $2 n$-dimensional closed symplectic manifold with a symplectic form $\omega$. We also equip $M$ with a Riemannian metric $g$.

Note that for technical reasons, we will sometimes assume that the symplectic manifold $(M, \omega)$ is of Lefschetz type. That is, the mapping

$$
\omega^{n-1}: H^{1}(M, \mathbb{R}) \rightarrow H^{2 n-1}(M, \mathbb{R}), \alpha \mapsto \alpha \wedge \omega^{n-1},
$$

is an isomorphism. The category of Lefschetz manifolds includes all Kähler manifolds, such has oriented surfaces and even dimensional tori.

\subsection{Harmonics 1-forms}

Let $H^{1}(M, \mathbb{R})$ denote the first de Rham cohomology group (with real coefficients) of $M$, and let $\mathcal{H}^{1}(M, g)$ denote the space of harmonic $1-$ forms on $M$ with respect to the Riemannian metric $g$. The set $\mathcal{H}^{1}(M, g)$ forms a finite dimensional vector space over $\mathbb{R}$ which is isomorphic to $H^{1}(M, \mathbb{R})$, and whose dimension is denoted $b_{1}(M)$, and called the first Betti number of the manifold $M$ [20. Taking $\left(h_{i}\right)_{1 \leq i \leq b_{1}(M)}$ as a basis of the vector space $\mathcal{H}^{1}(M, g)$, we equip $\mathcal{H}^{1}(M, g)$ with the Euclidean norm |.| defined as follows: for all $\mathrm{H} \in \mathcal{H}^{1}(\mathrm{M}, \mathrm{g})$ with

$$
H=\sum_{i=1}^{b_{1}(M)} \lambda_{i} h_{i},
$$

its norm is defined as

$$
|H|:=\sum_{i=1}^{b_{1}(M)}\left|\lambda_{i}\right| .
$$

We denote by $\mathcal{P} \mathcal{H}^{1}(M, g)$ the space of all smooth mappings $\mathcal{H}:[0,1] \rightarrow \mathcal{H}^{1}(M, g)$.

\section{On the classical symplectic dynamical systems}

\subsection{Symplectic diffeomorphisms and symplectic isotopies}

A diffeomorphism $\phi: M \rightarrow M$, is called symplectic if it preserves the symplectic form $\omega$, i.e. $\phi^{*}(\omega)=\omega$. We denote by $\operatorname{Symp}(M, \omega)$, the group of all symplectic diffeomorphisms of $(M, \omega)$. 
An isotopy $\left\{\phi_{t}\right\}$ of a symplectic manifold $(M, \omega)$ is said to be symplectic if $\phi_{t} \in \operatorname{Symp}(M, \omega)$ for each $t$, or equivalently, the vector field $\dot{\phi}_{\mathrm{t}}:=\frac{\mathrm{d} \phi_{\mathrm{t}}}{\mathrm{dt}} \circ \phi_{\mathrm{t}}^{-1}$ is symplectic for each $\mathrm{t}$. In particular, a symplectic isotopy $\left\{\psi_{t}\right\}$ is a Hamiltonian isotopy if for each $t$, the vector field $\dot{\psi}_{t}:=\frac{d \psi_{t}}{d t} \circ \psi_{t}^{-1}$ is Hamiltonian, i.e. there exists a smooth function $F:[0,1] \times M \rightarrow \mathbb{R}$, called generating Hamiltonian such that $\mathfrak{\imath}\left(\dot{\psi}_{t}\right) \omega=\mathrm{dF}_{\mathrm{t}}$, for each $\mathrm{t}$. Any Hamiltonian isotopy determines its generating Hamiltonian up to an additive constant. Throughout this paper we assume that every generating Hamiltonian $F:[0,1] \times M \rightarrow \mathbb{R}$ is normalized, i.e. we require that $\int_{M} F_{t} \omega^{n}=0$ for all $t$. Let $\mathcal{N}([0,1] \times M, \mathbb{R})$ denote the vector space of all smooth normalized Hamiltonians.

We denote by $\operatorname{Iso}(M, \omega)$ the group of all symplectic isotopies of $(M, \omega)$, and by $\operatorname{Symp}(M, \omega)$, the group of time-1 maps of all symplectic isotopies.

\subsection{Description of the classical symplectic isotopies}

We now recall the description of symplectic isotopies introduced in 5. Given any symplectic isotopy $\Phi=\left\{\phi_{t}\right\}$, one derives from Hodge's theory that the closed 1 -form $\mathfrak{\imath}\left(\dot{\phi}_{t}\right) \omega$ decomposes in a unique way as the sum of an exact $1-$ form $\mathrm{du}_{\mathrm{t}}^{\Phi}$ and a harmonic $1-$ form $\mathcal{H}_{\mathrm{t}}^{\Phi}$ [20]. Denote by $\mathrm{U}$ the normalized Hamiltonian of $\mathrm{U}^{\Phi}=\left(\mathrm{U}_{\mathrm{t}}^{\Phi}\right)$, and by $\mathcal{H}$ the smooth family of harmonic 1-forms $\mathcal{H}^{\Phi}=\left(\mathcal{H}_{\mathrm{t}}^{\Phi}\right)$. In [5] , the Cartesian product $\mathcal{N}([0,1] \times M, \mathbb{R}) \times \mathcal{P} \mathcal{H}^{1}(M, g)$ is denoted $\mathfrak{T}(\mathrm{M}, \omega, \mathrm{g})$, and equipped with a group structure which makes the bijection

$$
\mathfrak{A}: \operatorname{Iso}(M, \omega) \rightarrow \mathfrak{T}(M, \omega, g), \Phi \mapsto(\mathrm{U}, \mathcal{H})
$$

a group isomorphism. Under this identification, any symplectic isotopy $\Phi$ is denoted by $\phi_{(\mathrm{U}, \mathcal{H})}$ to mean that $\mathfrak{A}$ maps $\Phi$ onto $(\mathrm{U}, \mathcal{H})$, and the pair $(\mathrm{U}, \mathcal{H})$ is called the "generator" of the symplectic isotopy $\Phi$. For instance, a symplectic isotopy $\phi_{(0, \mathcal{H})}$, is a harmonic isotopy, and a symplectic isotopy $\phi_{(\mathrm{u}, 0)}$, is a Hamiltonian isotopy.

\subsection{Group structure on $\mathfrak{T}(M, \omega, g)$}

The product rule in $\mathfrak{T}(M, \omega, g)$ is given by,

$$
(\mathrm{U}, \mathcal{H}) \bowtie(\mathrm{V}, \mathcal{K})=\left(\mathrm{U}+\mathrm{V} \circ \phi_{(\mathrm{U}, \mathcal{H})}^{-1}+\widetilde{\Delta}\left(\mathcal{K}, \phi_{(\mathrm{U}, \mathcal{H})}^{-1}\right), \mathcal{H}+\mathcal{K}\right) .
$$

The inverse of $(\mathrm{U}, \mathcal{H})$, say $\overline{(\mathrm{U}, \mathcal{H})}$ is given by

$$
\overline{(\mathrm{U}, \mathcal{H})}=\left(-\mathrm{U} \circ \phi_{(\mathrm{U}, \mathcal{H})}-\widetilde{\Delta}\left(\mathcal{H}, \phi_{(\mathrm{U}, \mathcal{H})}\right),-\mathcal{H}\right) .
$$

In (3.2) and (3.3) the quantity $\widetilde{\Delta}$ is defined as follows: for any symplectic isotopy $\Psi=\left\{\psi^{t}\right\}$, and for any smooth family of closed 1 -forms $\alpha=\left(\alpha_{t}\right)$, we have

$$
\widetilde{\Delta}_{\mathrm{t}}(\alpha, \Psi)=\Delta_{\mathrm{t}}(\alpha, \Psi)-\frac{\int_{M} \Delta_{\mathrm{t}}(\alpha, \Psi) \omega^{\mathrm{n}}}{\int_{M} \omega^{\mathrm{n}}},
$$


where

$$
\Delta_{\mathrm{t}}(\alpha, \Psi):=\int_{0}^{\mathrm{t}} \alpha_{\mathrm{t}}\left(\dot{\psi}^{s}\right) \circ \psi^{\mathrm{s}} \mathrm{ds},
$$

for all $\mathrm{t}$ (see [5]). Also, it is proved in [16, 18] that $\Delta(\alpha, \Psi)$ is a 1 -cocycle.

\subsection{Metric structures on $\mathfrak{T}(M, \omega, g)$}

For all $(\mathrm{U}, \mathcal{H}),(\mathrm{V}, \mathcal{K}) \in \mathfrak{T}(M, \omega, \mathrm{g})$, set

$$
\mathrm{D}_{0}^{(1, \infty)}((\mathrm{U}, \mathcal{H}),(\mathrm{V}, \mathcal{K}))=\int_{0}^{1}\left[\left|\mathcal{H}_{\mathrm{t}}-\mathcal{K}_{\mathrm{t}}\right|+\operatorname{osc}\left(\mathrm{U}_{\mathrm{t}}-\mathrm{V}_{\mathrm{t}}\right)\right] \mathrm{dt}
$$

and

$$
\mathrm{D}_{0}^{\infty}((\mathrm{U}, \mathcal{H}),(\mathrm{V}, \mathcal{K}))=\max _{\mathrm{t}}\left[\left|\mathcal{H}_{\mathrm{t}}-\mathcal{K}_{\mathrm{t}}\right|+\operatorname{osc}\left(\mathrm{U}_{\mathrm{t}}-\mathrm{V}_{\mathrm{t}}\right)\right]
$$

where

$$
\operatorname{osc}(f)=\max _{x} f(x)-\min _{x} f(x),
$$

for all $f \in C^{\infty}(M, \mathbb{R})$. Therefore, the $\mathrm{L}^{\infty}$-Hofer-like metric and the $\mathrm{L}^{(1, \infty)}$-Hofer-like on $\mathfrak{T}(M, \omega, g)$ are defined respectively as follows:

$$
\mathrm{D}^{(1, \infty)}((\mathrm{U}, \mathcal{H}),(\mathrm{V}, \mathcal{K}))=\frac{\mathrm{D}_{0}^{(1, \infty)}((\mathrm{U}, \mathcal{H}),(\mathrm{V}, \mathcal{K}))+\mathrm{D}_{0}^{(1, \infty)}(\overline{(\mathrm{U}, \mathcal{H})}, \overline{(\mathrm{V}, \mathcal{K})})}{2}
$$

and

(see [2, 5]).

$$
\mathrm{D}^{\infty}((\mathrm{U}, \mathcal{H}),(\mathrm{V}, \mathcal{K}))=\frac{\mathrm{D}_{0}^{\infty}((\mathrm{U}, \mathcal{H}),(\mathrm{V}, \mathcal{K}))+\mathrm{D}_{0}^{\infty}(\overline{(\mathrm{U}, \mathcal{H})}, \overline{(\mathrm{V}, \mathcal{K})})}{2}
$$

\subsection{Displacement energy}

Definition 3.1. (4) The symplectic displacement energy es $(A)$ of a non empty set $A \subset M$ is:

$$
e_{S}(A)=\inf \left\{\|\phi\|_{H L} \mid \phi \in \operatorname{Symp}(M, \omega)_{0}, \phi(A) \cap A=\emptyset\right\} .
$$

Theorem 3.2. (目) For any non empty open set $\mathrm{A} \subset \mathrm{M}, \mathrm{e}_{\mathrm{S}}(\mathrm{A})$ is a strict positive number.

Note that in the definition of the displacement energy, the quantity $\|\cdot\|_{H L}$ stands for the usual Hofer-like norm defined in [2].

\section{On topological symplectic dynamical systems}

\subsection{The $\mathrm{C}^{0}$-topology}

Let Homeo $(M)$ be the group of all homeomorphisms of $M$ equipped with the $C^{0}$ - compact-open topology. This is the metric topology induced by the following distance

$$
d_{0}(f, h)=\max \left(d_{C^{\circ}}(f, h), d_{C^{\circ}}\left(f^{-1}, h^{-1}\right)\right),
$$


where $d_{C^{0}}(f, h)=\sup _{x \in M} d(h(x), f(x))$. On the space of all continuous paths $\lambda:[0,1] \rightarrow$ Homeo $(M)$ such that $\lambda(0)=i d_{M}$, we consider the $C^{0}$-topology as the metric topology induced by the metric

$$
\overline{\mathrm{d}}(\lambda, \mu)=\max _{t \in[0,1]} d_{0}(\lambda(t), \mu(t))
$$

\subsection{The origin of strong symplectic isotopies}

A result found in [15] (Corollary 3.7-[15], or more generally a result found in [16]) states that: Let $\Phi_{i}=\left\{\phi_{i}^{t}\right\}$ be a sequence of symplectic isotopies, $\Psi=\left\{\psi_{t}\right\}$ be another symplectic isotopy, and $\eta: t \mapsto \eta_{t}$ be a family of maps $\eta_{t}: M \rightarrow M$, such that the sequence $\Phi_{i}$ converges uniformly to $\eta$ and $l^{\infty}\left(\Psi^{-1} \circ \Phi_{i}\right) \rightarrow 0, i \rightarrow \infty$, then

$$
\eta=\Psi .
$$

Note that if, $\Psi$ generated by $(U, \mathcal{H})$ and $\Phi_{i}$ generated by $\left(U^{i}, \mathcal{H}^{i}\right)$, then replacing the condition

$$
l^{\infty}\left(\Psi^{-1} \circ \Phi_{i}\right) \rightarrow 0, i \rightarrow \infty
$$

by the condition

$$
\mathrm{D}^{\infty}\left((\mathrm{u}, \mathcal{H}),\left(\mathrm{u}^{\mathrm{i}}, \mathcal{H}^{\mathrm{i}}\right)\right) \rightarrow 0, i \rightarrow \infty
$$

does not break the result of Corollary 3.7-[15, 16]. Therefore, we can then ask the following question:

If in Corollary 3.7-[15, 16], the convergence $\mathrm{D}^{\infty}\left((\mathrm{U}, \mathcal{H}),\left(\mathrm{U}^{\mathrm{i}}, \mathcal{H}^{\mathrm{i}}\right)\right) \rightarrow 0, i \rightarrow \infty$, is replaced by the condition

$$
\mathrm{D}^{\infty}\left(\left(\mathrm{U}^{\mathrm{i}+1}, \mathcal{H}^{\mathrm{i}+1}\right),\left(\mathrm{U}^{i}, \mathcal{H}^{i}\right)\right) \rightarrow 0, i \rightarrow \infty,
$$

then what can we say about the geometries and the structures of the space of all such paths $\eta$ ?

The seek of a possible answer to the above question motivated the following definition:

Definition 4.1. ([6]) A continuous map $\xi:[0,1] \rightarrow$ Homeo $(M)$ with $\xi(0)=i d_{M}$, is called strong symplectic isotopy if there exists a $D^{\infty}$-Cauchy sequence $\left\{\left(F_{i}, \lambda_{i}\right)\right\} \subset \mathfrak{T}(M, \omega, g)$ such that $\overline{\mathrm{d}}\left(\phi_{\left(\mathrm{F}_{i}, \lambda_{i}\right)}, \xi\right) \rightarrow 0, i \rightarrow \infty$.

We denote by $\mathcal{P S S y m p e o}(M, \omega)$ the space of all strong symplectic isotopies. It is proved in [6. 14] that $\mathcal{P S S y m p e o}(M, \omega)$ is a group. If the manifold is simply connected, then the group $\mathcal{P S S y m p e o}(M, \omega)$ reduces to the group of continuous Hamiltonian flows [12]. The set of time-1 maps of all strong symplectic isotopies coincides with the group of all strong symplectic homeomorphisms, denoted here by $\operatorname{SSympeo}(M, \omega)$ (see [5, 3]). 


\subsection{Generators of ssympeotopies}

Let $\mathcal{N}^{0}([0,1] \times M, \mathbb{R})$ denotes the completion of the metric space $\mathcal{N}([0,1] \times M, \mathbb{R})$ with respect to the $\mathrm{L}^{\infty}$-Hofer norm, and let $\mathcal{P} \mathcal{H}^{1}(M, g)_{0}$ denotes the completion of the metric space $\mathcal{P} \mathcal{H}^{1}(M, g)$ with respect to the uniform sup norm. Consider the map

$$
\mathrm{J}^{\mathrm{O}}(\mathrm{M}, \omega, \mathrm{g}):=\mathcal{N}^{\mathrm{O}}([0,1] \times \mathrm{M}, \mathbb{R}) \times \mathcal{P} \mathcal{H}^{1}(\mathrm{M}, \mathrm{g})_{0},
$$

and the inclusion map

$$
i_{0}: \mathfrak{T}(M, \omega, g) \rightarrow \mathrm{J}^{0}(M, \omega, g) .
$$

This map is uniformly continuous with respect of the topology induced by the metric $\mathrm{D}^{\infty}$ on the space $\mathfrak{T}(M, \omega, g)$, and the natural topology of the complete metric space $\mathrm{J}^{0}(M, \omega, g)$. Now, let $\mathrm{L}(M, \omega, g)$ denotes the image of $\mathfrak{T}(M, \omega, g)$ under $i_{0}$, and $\mathfrak{T}(M, \omega, g)_{0}$ be the closure of $\mathrm{L}(M, \omega, g)$ inside the complete metric space $\mathrm{J}^{\mathrm{O}}(\mathrm{M}, \omega, \mathrm{g})$.

That is, $\mathfrak{T}(M, \omega, g)_{0}$ consists of pairs $(\mathrm{U}, \mathcal{H})$ where the mappings $(t, x) \mapsto \mathrm{U}_{\mathrm{t}}(\mathrm{x})$ and $\mathrm{t} \mapsto \mathcal{H}_{\mathrm{t}}$ are continuous, and for each $t, \mathcal{H}_{t}$ lies in $\mathcal{H}^{1}(M, g)$ such that there exists a $D^{\infty}$-Cauchy sequence $\left(\mathrm{U}^{i}, \mathcal{H}^{i}\right) \subset \mathfrak{T}(M, \omega, g)$ that converges to $(\mathrm{U}, \mathcal{H}) \in \mathrm{J}^{\mathcal{O}}(\mathrm{M}, \omega, g)$. Note that the sequence $\left(\mathrm{F}_{j}, \lambda_{j}\right)$ in definition (4.1) converges necessarily in the complete metric space $\mathfrak{T}(M, \omega, g)_{0}$. The latter limit is called the "generator" of strong symplectic isotopy (see [] $)$. We will often write $\left(F^{i}, \lambda^{i}\right) \stackrel{L^{\infty}}{\longrightarrow}(F, \lambda)$ to mean that the sequence $\left(F^{i}, \lambda^{i}\right)$ converges to $(F, \lambda)$ in the space $J^{0}(M, \omega, g)$.

Definition 4.2. ([6] $)$ The set GSSympeo $(M, \omega, g)$ is defined as the space of all the pairs $(\xi,(U, \mathcal{H}))$ where $\xi$ is a strong symplectic isotopy generated $(\mathrm{U}, \mathcal{H})$.

\section{Group structure on the space $\operatorname{GSSympeo}(M, \omega, g)$}

For all $(\xi,(F, \lambda)),(\mu,(V, \theta)) \in \operatorname{GSSympeo}(M, \omega, g)$, their product is given by,

$$
(\xi,(F, \lambda)) *(\mu,(V, \theta))=\left(\xi \circ \mu,\left(F+V \circ \xi^{-1}+\Delta^{0}\left(\theta, \xi^{-1}\right), \lambda+\theta\right)\right),
$$

and the inverse of the element $(\xi,(F, \lambda))$ is given by,

$$
\overline{(\xi,(F, \lambda))}=\left(\xi^{-1},\left(-F \circ \xi-\Delta^{0}(\lambda, \xi),-\lambda\right)\right),
$$

with

$$
\begin{aligned}
\Delta^{0}\left(\theta, \xi^{-1}\right) & :=\lim _{L^{\infty}}\left(\widetilde{\Delta}\left(\theta^{i}, \phi_{\left(F^{i}, \lambda^{i}\right)}^{-1}\right),\right. \\
\Delta^{0}(\lambda, \xi) & :=\lim _{L^{\infty}}\left(\widetilde{\Delta}\left(\lambda^{i}, \phi_{\left(F^{i}, \lambda^{i}\right.}\right)\right),
\end{aligned}
$$

where $\left(F^{i}, \lambda^{i}\right)$, and $\left(V^{i}, \theta^{i}\right)$ are two arbitrary sequences in $\mathfrak{T}(M, \omega, g)$ such that

$$
\left(F^{i}, \lambda^{i}\right) \stackrel{L^{\infty}}{\longrightarrow}(F, \lambda), \quad \phi_{\left(F^{i}, \lambda^{i}\right)} \stackrel{\bar{d}}{\rightarrow} \xi,
$$

and

$$
\left(V^{i}, \theta^{i}\right) \stackrel{L^{\infty}}{\longrightarrow}(V, \theta), \quad \phi_{\left(V^{i}, \theta^{i}\right)} \stackrel{\bar{d}}{\longrightarrow} \mu,
$$


with $\widetilde{\Delta}_{t}\left(\lambda^{i}, \phi_{\left(F^{i}, \lambda^{i}\right)}^{-1}\right)$ the normalized function of $\Delta_{t}\left(\lambda^{i}, \phi_{\left(F^{i}, \lambda^{i}\right)}^{-1}\right)$.

This set is known as a topological group with respect to the symplectic topology []. The symplectic topology on the space $\operatorname{GSSympeo}(M, \omega, g)$ is defined to be the subspace topology induced by the inclusion of the latter in the complete topological space $\mathcal{P}(\operatorname{Homeo}(M), i d) \times \mathfrak{T}(M, \omega, g)_{0}$.

\section{Question (a)}

Let $\left(M_{i}, \omega_{i}\right)$ be two closed symplectic manifolds equipped with two Riemannian metrics $g_{i}$, for $i=1,2$. If the group $\mathfrak{T}\left(M_{1}, \omega_{1}, g_{1}\right)_{0}$ is isomorphic to the group $\mathfrak{T}\left(M_{2}, \omega_{2}, g_{2}\right)_{0}$, then what can we say about: The manifolds $M_{1}$ and $M_{2}$ ? The symplectic structures $\omega_{1}$ and $\omega_{2}$ ? The Riemannian structures $g_{1}$ and $g_{2}$ ?

The following uniqueness results show that there is a bijective correspondence between the group of strong symplectic isotopies and that of their generators.

Theorem 4.3. ([] $[6])$ Let $(\mathrm{M}, \omega)$ be a Lefschetz closed symplectic manifold. Any strong symplectic isotopy determines a unique generator.

In the presence of a positive symplectic displacement energy from Banyaga-Hurtubise-Spaeth [4, we point out the following converse of Theorem 4.3, which in the same time gives the symplectic analogue of a result prove by $\mathrm{Oh}[12$.

Theorem 4.4. Any generator corresponds to a unique strong symplectic isotopy, i.e. if $(\gamma,(\mathrm{U}, \mathcal{H})),(\xi,(\mathrm{U}, \mathcal{H})) \in$ $\operatorname{GSSympeo}(M, \omega, g)$, then we must have $\gamma=\xi$.

Proof. Let $(\gamma,(\mathrm{U}, \mathcal{H}))$ and $(\xi,(\mathrm{U}, \mathcal{H}))$ be two elements of $\mathrm{GSSympeo}(\mathrm{M}, \omega, \mathrm{g})$. By definition of the group GSSympeo( $(M, \omega, g)$, there exist two sequences of symplectic isotopies $\phi_{\left(U_{i}, H_{i}\right)}$ and $\phi\left(V_{i}, K_{i}\right)$ such that:

$$
\phi_{\left(\mathrm{U}^{i}, \mathrm{H}^{i}\right)} \stackrel{\overline{\mathrm{d}}}{\rightarrow} \xi, \quad\left(\mathrm{U}^{\mathrm{i}}, \mathrm{H}^{\mathrm{i}}\right) \stackrel{\mathrm{L}^{\infty}}{\longrightarrow}(\mathrm{U}, \mathcal{H}),
$$

and

$$
\phi_{\left(\mathrm{V}^{i}, \mathrm{~K}^{\mathrm{i}}\right)} \stackrel{\overline{\mathrm{d}}}{\rightarrow} \gamma, \quad\left(\mathrm{V}^{\mathrm{i}}, \mathrm{K}^{\mathrm{i}}\right) \stackrel{\mathrm{L}^{\infty}}{\longrightarrow}(\mathrm{U}, \mathcal{H}) .
$$

Assume that $\gamma \neq \xi$, i.e. there exists $\left.\left.s_{0} \in\right] 0,1\right]$ such that $\gamma\left(s_{0}\right) \neq \xi\left(s_{0}\right)$. Since the map $\gamma^{-1}\left(s_{0}\right) \circ \xi\left(s_{0}\right)$ belongs to $\operatorname{Homeo}(M)$, then we derive from the identity $\gamma^{-1}\left(s_{0}\right) \circ \xi\left(s_{0}\right) \neq i d$, that there exists of a closed ball B which is entirely moved by $\gamma^{-1}\left(s_{0}\right) \circ \xi\left(s_{0}\right)$. From the compactness of B, and the uniform convergence of the sequence $\phi_{\left(\mathrm{U}_{i}, \mathrm{H}_{i}\right)}^{-1} \circ \phi_{\left(\mathrm{V}_{i}, \mathrm{~K}_{i}\right)}$ to $\gamma^{-1} \circ \xi$, we derive that

$$
\left(\phi_{\left(\mathrm{U}_{i}, \mathrm{H}_{i}\right)}^{-\mathrm{s}_{0}} \circ \phi_{\left(\mathrm{V}_{i}, \mathrm{~K}_{i}\right)}^{\mathrm{s}_{0}}\right)(\mathrm{B}) \cap(\mathrm{B})=\emptyset
$$


for all sufficiently large i. Relation (4.3) implies that,

$$
0<e_{S}(B) \leq l^{\infty}\left(\phi_{\left(U_{i}, H_{i}\right)}^{-1} \circ \phi\left(v_{i}, K_{i}\right)\right),
$$

for all sufficiently large $i$, where $e_{S}$ is the symplectic displacement energy from Banyaga-HurtubiseSpaeth 4], and $l^{\infty}$ (.) represents the $\mathrm{L}^{\infty}$-length functional for symplectic isotopies [2]. On the other hand, compute

$$
\begin{aligned}
& l^{\infty}\left(\phi_{\left(\mathrm{U}_{i}, \mathrm{H}_{i}\right)}^{-1} \circ \phi_{\left(\mathrm{V}_{i}, \mathrm{~K}_{\mathrm{i}}\right)}\right)=\max _{\mathrm{t}} \mathrm{osc}\left(-\mathrm{U}_{\mathrm{i}} \circ \phi_{\left(\mathrm{U}_{i}, \mathrm{H}_{i}\right)}+\mathrm{V}_{\mathrm{i}} \circ \phi_{\left(\mathrm{U}_{i}, \mathrm{H}_{i}\right)}+\widetilde{\Delta}_{\mathrm{t}}\left(\mathrm{K}_{\mathrm{i}}-\mathrm{H}_{\mathrm{i}}, \phi_{\left(\mathrm{U}_{i}, \mathrm{H}_{i}\right)}\right)\right) \\
& +\max _{t}\left|H_{i}^{t}-K_{i}^{t}\right| \\
& \leq \max _{\mathrm{t}}\left|\mathrm{H}_{i}^{\mathrm{t}}-\mathrm{K}_{i}^{\mathrm{t}}\right|+\max _{\mathrm{t}} \operatorname{osc}\left(\widetilde{\Delta}_{\mathrm{t}}\left(\mathrm{K}_{\mathrm{i}}-\mathrm{H}_{i}, \phi_{\left(\mathrm{U}_{i}, \mathrm{H}_{i}\right)}\right)\right) \\
& +\max _{t} \operatorname{osc}\left(-\mathrm{U}_{\mathrm{i}} \circ \phi_{\left(\mathrm{U}_{i}, \mathrm{H}_{i}\right)}+\mathrm{U}_{\mathrm{i}} \circ \phi_{\left(\mathrm{U}_{i+1}, \mathrm{H}_{i+1}\right)}\right) \\
& +\max _{\mathrm{t}} \mathrm{osc}\left(-\mathrm{U}_{\mathrm{i}} \circ \phi_{\left(\mathrm{U}_{i+1}, \mathrm{H}_{i+1}\right)}+\mathrm{U} \circ \phi_{\left(\mathrm{U}_{i+1}, \mathrm{H}_{i+1}\right)}\right) \\
& +\max _{\mathrm{t}} \operatorname{osc}\left(-\mathrm{U} \circ \phi_{\left(\mathrm{U}_{i+1}, \mathrm{H}_{\mathrm{i}+1}\right)}+\mathrm{V}_{\mathrm{i}} \circ \phi_{\left(\mathrm{U}_{i+1}, \mathrm{H}_{i+1}\right)}\right) \\
& +\max _{\mathrm{t}} \mathrm{osc}\left(-\mathrm{V}_{\mathrm{i}} \circ \phi_{\left(\mathrm{U}_{\mathrm{i}+1}, \mathrm{H}_{\mathrm{i}+1}\right)}+\mathrm{V}_{\mathrm{i}} \circ \phi_{\left(\mathrm{U}_{i}, \mathrm{H}_{\mathrm{i}}\right)}\right) \text {. }
\end{aligned}
$$

So, to prove that the right-hand side of the above estimates tends to zero when $i$ goes at infinity, we only need to prove that

$$
\max _{\mathrm{t}} \operatorname{osc}\left(\widetilde{\Delta}_{\mathrm{t}}\left(\mathrm{K}_{\mathrm{i}}-\mathrm{H}_{i}, \phi_{\left(\mathrm{U}_{i}, \mathrm{H}_{i}\right)}\right)\right) \rightarrow 0, i \rightarrow \infty .
$$

This follows from a straightforward application of Lemma 3.4 found in [5], or also from the inequality (2.15) found in Section 2.5 of [15] (or [16] for further generalization). This contradicts the positivity of the symplectic displacement energy in (4.4). This achieves the proof. $\square$

Based on Theorem 4.3 and Theorem 4.4, we will denote any strong symplectic isotopy $\lambda$ by $\lambda_{(\mathrm{u}, \mathcal{H})}$ to mean that it is generated by $(\mathrm{U}, \mathcal{H})$, or equivalently, to mean that $\lambda_{(\mathrm{u}, \mathcal{H})}$ is the limit of a sequence of symplectic isotopies $\phi_{\left(U^{i}, \mathcal{H}^{i}\right)}$ with respect to the metric $\left(\overline{\mathrm{d}}+\mathrm{D}^{\infty}\right)$, i.e.

$$
\lambda_{(\mathrm{U}, \mathcal{H})}=\lim _{\mathrm{C}^{0}+\mathrm{L}^{\infty}}\left(\phi_{\left(\mathrm{U}^{i}, \mathcal{H}^{i}\right)}\right) .
$$

The following result shows that any strong symplectic isotopy which is a 1-parameter group decomposes as composition of a smooth harmonic flow and a continuous Hamiltonian flow.

Theorem 4.5. Let $\beta=\left(\beta_{\mathrm{t}}\right)_{\mathrm{t} \in[0,1]}$ be a strong symplectic isotopy. Assume that $\beta=\left(\beta_{\mathrm{t}}\right)_{\mathrm{t} \in[0,1]}$ is a 1 -parameter group i.e:

$$
\beta_{\mathrm{t}+\mathrm{s}}=\beta_{\mathrm{t}} \circ \beta_{\mathrm{s}},
$$

$\forall \mathrm{s}, \mathrm{t} \in[0,1]$ such that $(\mathrm{s}+\mathrm{t})$ lies in $[0,1]$. Then, its generator $(\mathrm{F}, \lambda)$ is time-independent, $\lambda$ is a smooth harmonic 1-form, and $\mathrm{F}$ is a continuous function. 
Proof. Let $\beta_{(\mathrm{F}, \lambda)}$ be a strong symplectic isotopy. Assume that

$$
\beta_{(F, \lambda)}^{t+s}=\beta_{(F, \lambda)}^{t} \circ \beta_{(F, \lambda)}^{s},
$$

$\forall s, t \in[0,1]$ such that $(s+t)$ lies in $[0,1]$. This, implies that, for all $s, t \in[0,1]$ such that $(s+t)$ lies in $[0,1]$, we have

$$
\beta_{(F, \lambda)}^{t}=\beta_{(F, \lambda)}^{s+t} \circ\left(\beta_{(F, \lambda)}^{s}\right)^{-1} .
$$

We may prove that $\lambda^{t}=\lambda^{s}$, and $F^{t}(x)=F^{s}(x)$ for all $t, s \in[0,1]$, and for all $x \in M$. By definition of the path $\beta_{(F, \lambda)}$, we have

$$
\beta_{(F, \lambda)}=\lim _{C^{0}+L^{\infty}}\left(\phi_{\left(F_{i}, \lambda_{i}\right)}\right),
$$

where $\phi_{\left(F_{i}, \lambda_{i}\right)}$ is a sequence of symplectic isotopies. Then, one can check that for each fixed $s \in[0,1]$, the sequence of symplectic maps defined by

$$
\Psi_{s}^{i}(t):=\phi_{\left(F_{i}, \lambda_{i}\right)}^{(t+s)} \circ\left(\phi_{\left(F_{i}, \lambda_{i}\right)}^{s}\right)^{-1}
$$

for all $t$ such that $(t+s)$ belongs to $[0,1]$, converges uniformly to $\beta_{(F, \lambda)}$.

Now, for each $i$ compute the derivative (in $t$ ) of the path $t \mapsto \Psi_{s}^{i}(t)$, and derive from the chain rule that at each time $t$, the tangent vector to the path $t \mapsto \Psi_{s}^{i}(t)$ coincides with the tangent vector to the path $t \mapsto \phi_{\left(F_{i}, \lambda_{i}\right)}^{(t+s)}$. That is, the isotopy $t \mapsto \Psi_{s}^{i}(t)$ is generated by an element $\left(U_{s}^{i}, H_{s}^{i}\right)$ where

$$
\mathrm{U}_{\mathrm{s}}^{\mathrm{i}}(\mathrm{t})=\mathrm{F}_{\mathrm{i}}^{\mathrm{t}+\mathrm{s}}
$$

and

$$
H_{s}^{i}(t)=\lambda_{i}^{t+s},
$$

for all $t$ such that $(t+s)$ belongs to $[0,1]$, and for each $i$. Furthermore, the sequence of generators $\left(U_{s}^{i}, H_{s}^{i}\right)$ converges in the $L^{\infty}-$ metric to $\left(U_{s}, H_{s}\right)$ where

$$
\mathrm{U}_{\mathrm{s}}(\mathrm{t})=\mathrm{F}^{\mathrm{t}+\mathrm{s}}
$$

and

$$
H_{s}(t)=\lambda^{t+s}
$$

for all $t$ such that $(t+s)$ belongs to $[0,1]$. Therefore, we have proved that for each $s$,

$$
\left(\left\{\Psi_{s}^{i}(t)\right\}_{t},\left(U_{s}^{i}, H_{s}^{i}\right)\right) \stackrel{C^{0}+L^{\infty}}{\longrightarrow}\left(\beta_{(F, \lambda)},\left(U_{s}, H_{s}\right)\right) .
$$

Thus, Theorem 4.3 tells us that for each fixed $s \in[0,1]$ we must have $\lambda^{t}=\lambda^{t+s}$, and $F^{t}(x)=F^{s+t}(x)$ for all $t \in[0,1]$ such that $(t+s)$ belongs to $[0,1]$ and for all $x \in M$. Since this is always true for a given $s \in[0,1]$ such that $(t+s)$ belongs to $[0,1]$, we derive that $\lambda^{t}=\lambda^{0}$, and $F^{t}(x)=F^{0}(x)$ for all $\mathrm{t} \in[0,1]$, and for all $x \in M$. This achieves the proof.

We have the following fact. 
Lemma 4.6. Let $\lambda_{(\mathrm{u}, \mathcal{H})}$ be any strong symplectic isotopy. For each fixed $\mathrm{s} \in[0,1)$, the path $\mathrm{t} \mapsto \lambda_{(\mathrm{V}, \mathcal{K})}^{\mathrm{t}}:=\lambda_{(\mathrm{U}, \mathcal{H})}^{(\mathrm{t}+\mathrm{s})} \circ\left(\lambda_{(\mathrm{U}, \mathcal{H})}^{\mathrm{s}}\right)^{-1}$ is a strong symplectic isotopy generated by $(\mathrm{V}, \mathcal{K})$ where $\mathrm{V}(\mathrm{t}, \mathrm{x})=$ $\mathrm{U}(\mathrm{t}+\mathrm{s}, \mathrm{x})$, and $\mathcal{K}_{\mathrm{t}}=\mathcal{H}_{(\mathrm{t}+\mathrm{s})}$, for all $\mathrm{t} \in[0,1-\mathrm{s}]$, and for all $\mathrm{x} \in \mathrm{M}$.

Proof. Assume that $\lambda_{(\mathrm{U}, \mathcal{H})}=\lim _{\mathrm{C}^{0}+\mathrm{L}^{\infty}}\left(\phi_{\left(\mathrm{U}^{i}, \mathcal{H}^{\mathrm{i}}\right)}\right)$. For each fixed $s \in[0,1)$, consider the sequence $\left\{\phi_{\left(V^{i}, \mathcal{K}^{i}\right)}\right\}$ of symplectic isotopies defined by

$$
\phi_{\left(\mathrm{V}^{i}, \mathcal{K}^{i}\right)}^{\mathrm{t}}=\phi_{\left(\mathrm{U}^{i}, \mathcal{H}^{i}\right)}^{(\mathrm{t}+\mathrm{s})} \circ\left(\phi_{\left(\mathrm{U}^{i}, \mathcal{H}^{i}\right)}^{\mathrm{s}}\right)^{-1},
$$

for all $t \in[0,1-s]$, and for each $i$. Compute the derivative (in $t$ ) of the path $t \mapsto \phi_{\left(V^{i}, \mathcal{K}^{i}\right)}^{t}$, and derive from the chain rule that at each time $t$, the tangent vector to the path $t \mapsto \phi_{\left(V^{i}, \mathcal{K}^{i}\right)}^{t}$ coincides with the tangent vector to the path $t \mapsto \phi_{\left(U^{i}, \mathcal{H}^{i}\right)}^{(t+s)}$, or equivalently we get $V^{i}(t)=U^{i}(t+s)$, and $\mathcal{K}_{t}^{i}=\mathcal{H}_{(t+s)}^{i}$ for all $t \in[0,1-s]$. A straightforward computation implies that the sequence of symplectic isotopies $\mathrm{t} \mapsto \phi_{\left(V^{i}, \mathcal{K}^{i}\right)}^{\mathrm{t}}$ converges in $\overline{\mathrm{d}}$ to $\lambda_{(\mathrm{U}, \mathcal{H})}^{(\mathrm{t}+\mathrm{s})} \circ\left(\lambda_{(\mathrm{U}, \mathcal{H})}^{\mathrm{s}}\right)^{-1}$, as well as the sequence of generators $\left(V^{i}, \mathcal{K}^{i}\right)$ converges in the $L^{\infty}$-topology to an element $(\mathrm{V}, \mathcal{K})$ such that $\mathrm{V}(\mathrm{t}, \mathrm{x})=\mathrm{U}(\mathrm{t}+\mathrm{s}, \mathrm{x})$, and $\mathcal{K}_{t}=\mathcal{H}_{(t+s)}$ for all $t \in[0,1-s]$, and for all $x \in M$. That is, $\lambda_{(V, \mathcal{K})}$ is a strong symplectic isotopy generated by $(\mathrm{V}, \mathcal{K})$. This completes the proof.

Theorem 4.5 implies that any strong symplectic isotopy which is a 1-parameter group decomposes as the composition of smooth harmonic flow and a continuous Hamiltonian flow in the sense of Oh-Müller [13].

\section{Question (b)}

Is 1-parameter group any strong symplectic isotopy which decomposes into the composition of smooth harmonic flow and a continuous Hamiltonian flow?

\section{Topological Hofer-like geometry}

\section{Concatenation of strong symplectic isotopies}

Let $0<\mathfrak{u}_{1}<\mathfrak{u}_{2}<1$. For each $0<\epsilon<\mathfrak{u}_{1}$ such that $\mathfrak{u}_{2} \leq 1-\epsilon<1$, pick a smooth increasing function $a:[0,1] \rightarrow[0,1]$, such that a restricted to $[0, \epsilon]$ (resp. $[(1-\epsilon), 1])$ is equal to 0 (resp. 1). Then define two smooth functions as follows:

$$
\left\{\begin{array}{l}
\lambda(t)=a(2 t), \quad 0 \leq t \leq \frac{1}{2} \\
\mu(t)=a(2 t-1), \quad \frac{1}{2} \leq t \leq 1 .
\end{array}\right.
$$

Now, given two strong symplectic isotopies $\gamma=\gamma_{(\mathrm{U}, \mathcal{H})}$ and $\beta=\beta_{(\mathrm{V}, \mathcal{K})}$, one defines the left concatenation $\gamma *_{\imath} \beta$ of $\beta$ by $\gamma$ as follows;

$$
\left(\gamma *_{l} \mu\right)(t)=\left\{\begin{array}{l}
\gamma^{\lambda(t)}, \text { if } 0 \leq t \leq \frac{1}{2}, \\
\gamma^{1} \circ \beta_{\mu(t)}, \text { if } \frac{1}{2} \leq t \leq 1 .
\end{array}\right.
$$


The path $\gamma *_{l} \mu$ is a strong symplectic isotopy generated by an element $\left(F_{l}, \theta_{l}\right)$ given by:

$$
\left(F_{l}, \theta_{l}\right)(t)=\left\{\begin{array}{l}
\dot{\lambda}(t)\left(U^{\lambda(t)}, \mathcal{H}^{\lambda(t)}\right), \quad \text { if } 0 \leq t \leq \frac{1}{2}, \\
\dot{\mu}(t)\left(V^{\mu(t)} \circ \gamma^{-1}+\square_{\mu(t)}(\gamma, \mathcal{K}), \mathcal{K}^{\mu(t)}\right) \text { if } \frac{1}{2} \leq t \leq 1,
\end{array}\right.
$$

so that if

$$
\left(\mathrm{V}^{\mathrm{i}}, \mathcal{K}^{\mathrm{i}}\right) \stackrel{\mathrm{L}^{\infty}}{\longrightarrow}(\mathrm{V}, \mathcal{K}), \text { and } \phi_{\left(\mathrm{V}^{\mathrm{i}}, \mathcal{K}^{\mathrm{i}}\right)} \stackrel{\overline{\mathrm{d}}}{\rightarrow} \beta
$$

and

$$
\left(\mathrm{U}^{\mathrm{i}}, \mathcal{H}^{\mathrm{i}}\right) \stackrel{\mathrm{L}^{\infty}}{\longrightarrow}(\mathrm{U}, \mathcal{H}) \text {, and } \Phi_{\mathrm{i}}=\phi_{\left(\mathrm{U}^{i}, \mathcal{H}^{i}\right)} \stackrel{\overline{\mathrm{d}}}{\rightarrow} \gamma
$$

then

$$
\square_{\mathrm{t}}(\gamma, \mathcal{K})=\lim _{\mathrm{L}^{\infty}}\left(\int_{0}^{1} \mathcal{K}_{\mathrm{t}}^{\mathrm{i}}\left(\dot{\Phi}_{i}(\mathrm{~s})\right) \circ \Phi_{\mathfrak{i}}(\mathrm{s}) \mathrm{ds}\right),
$$

for each $t \in[0,1]$. The existence of the limit in (5.1) is supported by results found in [15, 16] (see Lemma 3.10-15 and Section 2.5-[16]). Note that the path $\gamma *_{\imath} \beta$ is a strong symplectic isotopy from the identity to $\gamma^{1} \circ \beta^{1}$.

Similarly, one can define the right concatenation $\beta *_{r} \gamma$ of $\beta$ by $\gamma$ as follows;

$$
\left(\beta *_{r} \gamma\right)(t)=\left\{\begin{array}{l}
\beta^{\lambda(t)}, \text { if } 0 \leq t \leq \frac{1}{2} \\
\gamma_{\mu(t)} \circ \beta^{1}, \text { if } \frac{1}{2} \leq t \leq 1
\end{array}\right.
$$

The path $\beta *_{r} \gamma$ is a strong symplectic isotopy from the identity to $\beta^{1} \circ \gamma^{1}$, generated by an element $\left(\mathrm{G}_{\mathrm{r}}, \Xi_{\mathrm{r}}\right)$ given by:

$$
\left(G_{r}, \Xi_{r}\right)(t)=\left\{\begin{array}{l}
\dot{\lambda}(t)\left(V^{\lambda(t)}, \mathcal{K}^{\lambda(t)}\right), \quad \text { if } 0 \leq t \leq \frac{1}{2} \\
\dot{\mu}(t)\left(U^{\mu(t)}, \mathcal{H}^{\mu(t)}\right), \quad \text { if } \frac{1}{2} \leq t \leq 1
\end{array}\right.
$$

\subsection{Length of strong symplectic isotopies}

For any strong symplectic isotopy $\gamma_{(\mathrm{U}, \mathcal{H})}$, we define its $\mathrm{L}^{\infty}$-length and $\mathrm{L}^{(1, \infty)}$-length respectively by

$$
l_{\infty}\left(\gamma_{(\mathrm{u}, \mathcal{H})}\right)=\max _{\mathrm{t}}\left(\operatorname{osc}\left(\mathrm{U}_{\mathrm{t}}\right)+\left|\mathcal{H}_{\mathrm{t}}\right|\right)
$$

and

$$
\mathrm{l}_{(1, \infty)}\left(\gamma_{(\mathrm{U}, \mathcal{H})}\right)=\int_{0}^{1}\left(\operatorname{osc}\left(\mathrm{U}_{\mathrm{t}}\right)+\left|\mathcal{H}_{\mathrm{t}}\right|\right) \mathrm{dt} .
$$

This agrees with the usual definition of Hofer-like lengths of symplectic isotopies [2]: If $\gamma_{(\mathrm{U}, \mathcal{H})}$ is a smooth isotopy, then above lengths coincide with the usual Hofer-like lengths. If the manifold $M$ is simple connected, then the above length reduces to the length of continuous Hamiltonian flows defined by Oh [12. Furthermore, we can as well equip $\mathcal{P} \operatorname{SSympeo}(M, \omega)$ with a metric $\overline{\mathrm{D}^{\infty}}$ which is the topological analogue of the metric $\mathrm{D}^{\infty}$. On the other hand, by writing $\overline{\mathrm{d}}$ as the metric that 
induces the $\mathrm{C}^{0}-$ compact open topology, we shall call the metric topology induced by $\overline{\mathrm{d}}+\overline{\mathrm{D}^{\infty}}$ on $\mathcal{P S S y m p e o}(M, \omega)$, the symplectic topology on it.

Remark 5.1. If we consider the right concatenation $\beta *_{r} \gamma$ of $\beta$ by $\gamma$, then

$$
l_{(1, \infty)}\left(\beta *_{r} \gamma\right)=l_{(1, \infty)}(\beta)+l_{(1, \infty)}(\gamma)
$$

But, in general, we do not know whether we always have

$$
l_{\infty}\left(\beta *_{r} \gamma\right) \leq l_{\infty}(\beta)+l_{\infty}(\gamma)
$$

or not. However, using the fundamental lemma of Hofer-like geometry (see Lemma 3.9- 16, 15]), one can show that for all $\epsilon>0$, there exists a strong symplectic isotopy $\eta$ with the same extremities that $\beta *_{r} \gamma$, such that

$$
l_{\infty}(\eta)<l_{(1, \infty)}\left(\beta *_{r} \gamma\right)+\epsilon \leq l_{\infty}(\beta)+l_{\infty}(\gamma)+\epsilon
$$

\subsection{Hofer-like metric for strong symplectic homeomorphisms}

As in 2, using the above definitions of lengths, we can assign to each strong symplectic homeomorphism two real numbers called "energies" defined as follows: pick any $h \in \operatorname{SSympeo}(M, \omega)$, denote by $\boldsymbol{\Delta}(\mathrm{h})$ the set of all strong symplectic isotopies $\gamma_{(\mathrm{u}, \mathcal{H})}$ with time-1 map $\mathrm{h}$, i.e.,

$$
\mathbf{\Delta}(\mathrm{h}):=\{\Phi \in \mathcal{P} \operatorname{SSympeo}(M, \omega): \Phi(1)=h\}
$$

Then assign to $h$ the real numbers $\bar{e}(h)$, and $\bar{e}_{0}(h)$ defined respectively as follows:

$$
\bar{e}(h)=\inf _{\Phi \in \mathbf{\Lambda}(\mathrm{h})}\left\{l_{\infty}(\Phi)\right\}
$$

and

$$
\bar{e}_{0}(h)=\inf _{\Phi \in \mathbf{\Lambda}(h)}\left\{l_{(1, \infty)}(\Phi)\right\}
$$

Using the above definitions of energies, we define two real valued functions on SSympeo(M, $\omega)$ as follows: for each $h \in \operatorname{SSympeo}(M, \omega)$, we have

$$
\|\mathrm{h}\|_{S H L}=\frac{\bar{e}(h)+\bar{e}\left(h^{-1}\right)}{2}
$$

and

$$
\|h\|_{S H L}^{(1, \infty)}=\frac{\bar{e}_{0}(h)+\bar{e}_{0}\left(h^{-1}\right)}{2} .
$$

We have the following properties.

Theorem 5.2. Let $(\mathrm{M}, \omega)$ be any Lefschetz closed symplectic manifold. Given two strong symplectic homeomorphisms $\mathrm{h}$, and $\mathrm{f}$ we have : 
(1) $\|\mathrm{h}\|_{\mathrm{SHL}}=0$, if and only if, $\mathrm{h}=\mathrm{id} \mathrm{d}_{\mathrm{M}}$,

(2) $\|\mathrm{h}\|_{S H L}=\left\|h^{-1}\right\|_{S H L}$,

(3) $\|\mathrm{h} \circ \mathrm{f}\|_{S H L} \leq\|\mathrm{h}\|_{S H L}+\|f\|_{S H L}$.

Proof. The item (2) follows from the definition of the map $\left\|_{,}\right\|_{S H L}$. For (1), we adapt the proof of the nondegeneracy of the Hofer norm for Hamiltonian homeomorphism given by Oh [12]: Suppose that $h \neq i d_{M}$. Then $h$ displaces a small nonempty compact ball B with positive symplectic displacement energy $e_{S}(B)>0$ (we refer to [4] for the definition of symplectic displacement energy). For such a ball $B$, we set $\delta=e_{S}(B)$. The characterization of the infimum tells us that one can find a strong symplectic isotopy $\gamma_{(\mathrm{U}, \mathcal{H})}^{\delta}$ with $\gamma_{(\mathrm{U}, \mathcal{H})}^{\delta}(1)=\mathrm{h}$, such that

$$
\|\mathrm{h}\|_{\mathrm{SHL}}>\mathrm{l}_{\infty}\left(\gamma_{(\mathrm{U}, \mathcal{H})}^{\delta}\right)-\frac{\delta}{4} .
$$

On the other hand, it follows from the definition of strong symplectic isotopies that there exists a sequence $\left(\phi_{\left(F_{i}, \lambda_{i}\right)}\right)$ that converges to $\gamma_{(\mathrm{U}, \mathcal{H})}$ with respect to the $\left(\mathrm{C}^{0}+\mathrm{L}^{\infty}\right)$-topology. So, we can find a larger integer $i_{0}$ for which the path $\phi_{\left(\mathrm{F}_{i_{0}}, \lambda_{i_{0}}\right)}$ is sufficiently close to $\gamma_{(\mathrm{U}, \mathcal{H})}\left[\operatorname{resp} . \phi_{\left(\mathrm{F}_{i_{0}}, \lambda_{i_{0}}\right)}^{-1}\right.$ sufficiently close to $\left.\gamma_{(\mathrm{U}, \mathcal{H})}^{-1}\right]$ with respect to the $\left(\mathrm{C}^{0}+\mathrm{L}^{\infty}\right)$-topology, and so that

$$
l_{\infty}\left(\gamma_{(\mathrm{U}, \mathcal{H})}^{\delta}\right)>l_{\infty}\left(\phi_{\left(\mathrm{F}_{i_{0}}, \lambda_{i_{0}}\right)}\right)-\frac{\delta}{4}
$$

where $\phi_{i_{0}}=\phi_{\left(\mathrm{F}_{i_{0}}, \lambda_{i_{0}}\right)}^{1}$ displaces B. It follows from the definition of Banyaga's Hofer-like norm $\|,\|_{H L}\left(\right.$ see [2]) that $l_{\infty}\left(\phi_{\left(\mathrm{F}_{i_{0}}, \lambda_{i_{0}}\right)}\right) \geq\left\|\phi_{i_{0}}\right\|_{H L}$, i.e.

$$
l_{\infty}\left(\phi_{\left(\mathrm{F}_{i_{0}}, \lambda_{i_{0}}\right)}\right)-\frac{2 \delta}{4} \geq\left\|\phi_{i_{0}}\right\|_{H L}-\frac{\delta}{2} .
$$

Then, we derive from the definition of symplectic displacement energy $e_{S}$ (see [4) that

$$
\left\|\phi_{i_{0}}\right\|_{H L}-\frac{\delta}{2} \geq e_{S}(B)-\frac{\delta}{2}=\delta-\frac{\delta}{2}=\frac{\delta}{2}>0 .
$$

Summarizing the above statements together gives,

$$
\|\mathrm{h}\|_{\mathrm{SHL}}>\mathrm{l}_{\infty}\left(\gamma_{(\mathrm{U}, \mathcal{H})}^{\delta}\right)-\frac{\delta}{4}>l_{\infty}\left(\phi_{\left(\mathrm{F}_{i_{0}}, \lambda_{i_{0}}\right)}\right)-\frac{2 \delta}{4} \geq\left\|\phi_{i_{0}}\right\|_{H L}-\frac{\delta}{2}=\frac{\delta}{2}>0 .
$$

For (3), let $\mathrm{h}$ and $\mathrm{f}$ be two strong symplectic homeomorphisms, pick $\gamma \in \mathbf{\Lambda}(\mathrm{h})$ and $\beta \in \mathbf{\Lambda}(\mathrm{f})$, and derive from Remark 5.1 that for all $\epsilon>0$, there exists a strong symplectic isotopy $\eta$ with the same extremities as the right concatenation $\gamma *_{r} \beta$ such that

$$
\bar{e}(h \circ f) \leq l_{\infty}(\eta)<l_{(1, \infty)}\left(\gamma *_{r} \beta\right)+\epsilon \leq l_{\infty}(\gamma)+l_{\infty}(\beta)+\epsilon,
$$

and passing to the infimum in (5.11) yields

$$
\bar{e}(h \circ f) \leq \bar{e}(h)+\bar{e}(f) .
$$


Similarly, one shows that

$$
\bar{e}\left(f^{-1} \circ h^{-1}\right) \leq \bar{e}\left(h^{-1}\right)+\bar{e}\left(f^{-1}\right) .
$$

Relations (5.12) and (5.13) imply

$$
\|\mathrm{h} \circ \mathrm{f}\|_{\mathrm{SHL}} \leq\|\mathrm{h}\|_{\mathrm{SHL}}+\|\mathrm{f}\|_{\mathrm{SHL}} \cdot
$$

This completes the proof.

Let SSympeo $(M, \omega)$ denote the group of strong symplectic homeomorphisms equipped with the symplectic topology (see [3, 5, 6] for more convenience).

Theorem 5.3. Let $(\mathrm{M}, \omega)$ be a closed symplectic manifold. Then, the mapping

$$
\|\cdot\|_{\text {SHL }}: \operatorname{SSympeo}(\mathrm{M}, \omega) \rightarrow \mathbb{R}, \mathrm{h} \mapsto\|\mathrm{h}\|_{\mathrm{SHL}}
$$

is continuous.

Proof. In the way that the symplectic topologies had been defined on the spaces SSympeo $(M, \omega)$ and $\mathcal{P S S y m p e o}(M, \omega)$ (see [5]), the map

$$
\begin{gathered}
\|.\|_{\text {SHL }}: \operatorname{SSympeo}(M, \omega) \rightarrow \mathbb{R}_{+}, \\
h \mapsto\|h\|_{\text {SHL }}
\end{gathered}
$$

is continuous if and only if, for any open subset $\mathcal{I}$ in $\mathbb{R}_{+}$, the set $\|\cdot\|_{S H L}^{-1}(\mathcal{I})$ is open in $\mathfrak{S} \mathfrak{S y m p e o}(M, \omega)$. But, considering the following evaluation map

$$
\begin{aligned}
\text { ev }: \mathcal{P S S y m p e o}(M, \omega) & \rightarrow \operatorname{SS} \operatorname{Sympeo}(M, \omega), \\
\gamma_{(\mathrm{u}, \mathcal{H})} & \mapsto \gamma(1),
\end{aligned}
$$

which is a continuous mapping with respect to the symplectic topology on $\mathcal{P S S y m p e o}(M, \omega)$. We see that $\|\cdot\|_{S H L}^{-1}(\mathcal{I})$ is an open subset in $\operatorname{SSympeo}(M, \omega)$ if and only if $e v^{-1}\left(\|\cdot\|_{S H L}^{-1}(\mathcal{I})\right)$ is an open set in $\mathcal{P S S y m p e o}(M, \omega)$. That is, the map

$$
\|\cdot\|_{\text {SHL }}: \operatorname{SSympeo}(M, \omega) \rightarrow \mathbb{R}_{+}
$$

is continuous if and only if the map

$$
\|\cdot\|_{\text {SHL }} \text { o ev }: \mathcal{P S S y m p e o}(M, \omega) \rightarrow \mathbb{R}_{+},
$$

is continuous with respect to the symplectic topology on the space $\mathcal{P S S y m p e o}(M, \omega)$. Then, the continuity of the map

$$
\mathfrak{S} \operatorname{Sympeo}(M, \omega) \rightarrow \mathbb{R}_{+}
$$




$$
\mathrm{h} \mapsto\|\mathrm{h}\|_{\text {SHL }},
$$

is proved as follows: Let $\left\{\gamma_{\left(\mathrm{U}_{k}, \mathcal{H}_{\mathrm{k}}\right)}\right\}$ be a sequence in $\mathcal{P S S y m p e o}(M, \omega)$ that converges to $\gamma_{(\mathrm{U}, \mathcal{H})} \in$ $\mathcal{P S S y m p e o}(M, \omega)$ with respect to the $\left(\mathrm{C}^{0}+\mathrm{L}^{\infty}\right)$-topology. Set $\gamma_{k}(1)=\gamma_{\left(\mathrm{U}_{k}, \mathcal{H}_{k}\right)}^{1}$, for each $k$, and $\gamma(1)=\gamma_{(\mathrm{U}, \mathcal{H})}^{1}$. Now, compute

$$
\left|\bar{e}(\gamma(1))-\bar{e}\left(\gamma_{k}(1)\right)\right| \leq \bar{e}\left(\left[\gamma_{\left(\mathrm{u}_{k}, \mathcal{H}_{k}\right)}^{1}\right]^{-1} \circ \gamma_{(\mathrm{u}, \mathcal{H})}^{1}\right) \leq l_{\infty}\left(\gamma_{\left(\mathrm{u}_{k}, \mathcal{H}_{\mathrm{k}}\right)}^{-1} \circ \gamma_{(\mathrm{u}, \mathcal{H})}\right) \rightarrow 0, \mathrm{k} \rightarrow \infty .
$$

This completes the proof.

The main result of this paper is the following theorem.

Theorem 5.4. (Topological Hofer-like norm) Let $(\mathrm{M}, \omega)$ be a closed Lefschetz symplectic manifold. Then, the map $\|,\|_{\mathrm{SHL}}$ induces a norm on the group $\operatorname{SSympeo}(\mathrm{M}, \omega)$.

Proof. The proof of Theorem 5.4 is a consequence of Theorem 5.2 and Theorem 5.3

Theorem 5.4] is the symplectic analogue of a result that was proved by Oh [12] for Hamiltonian homeomorphisms. In particular, if the manifold $M$ has a trivial first de Rham cohomology group $H^{1}(M, \mathbb{R})$, then the norm $\|\cdot\|_{S H L}$ reduces to the norm $\|\cdot\|_{\text {Oh }}$ constructed by Oh on the group of all Hamiltonian homeomorphisms [13, 12].

Note that the norm $\|,\|_{S H L}$ defined on the group $\operatorname{SSympeo}(M, \omega)$ induces a right-invariant distance on it, defined by:

$$
\mathfrak{D}(\phi, \psi)=\left\|\phi \circ \psi^{-1}\right\|_{\mathrm{SHL}}
$$

for all $\phi, \psi \in \operatorname{SSympeo}(M, \omega)$.

Note that when $H^{1}(M, \mathbb{R}) \neq 0$, it follows from the decomposition theorem of strong symplectic homeomorphisms that the group of Hamiltonian homeomorphisms is strictly contained in the group of strong symplectic homeomorphisms (see [14]). So, it is an interesting task to investigate whether the topology induced by the norm $\|\cdot\|_{S H L}$ is an extension of Oh's topology or not: This motivated the following result.

Theorem 5.5. (Topological Banyaga's conjecture) The norm $\|\cdot\|_{S H L}$ restricted to the group of Hamiltonian homeomorphisms is equivalent to Oh's norm: There exists a positive constant $\mathrm{k}>0$, such that

$$
\|\phi\|_{\text {SHL }} \leq\|\phi\|_{\text {Oh }} \leq \kappa\|\phi\|_{S H L},
$$

for all Hamiltonian homeomorphism $\phi$.

This result is motivated in party by a conjecture which can be found in [2]. The latter conjecture first was proved by Buss-Leclercq [7, and an alternate proof of the same conjecture is given 
in [17. This conjecture stated that the restriction of Banyaga's Hofer-like norm to the group of Hamiltonian diffeomorphisms is equivalent to Hofer's norm.

Proof of Theorem [5.5. By construction, we always have

$$
\|\cdot\|_{\text {SHL }} \leq\|\cdot\|_{\mathrm{Oh}}
$$

To complete the proof, we need to show that there exists a positive finite constant $\mathrm{k}$ such that

$$
\|\cdot\| \mathrm{Oh} \leq \mathrm{K}\|\cdot\|_{\mathrm{SHL}}
$$

or equivalently, via the sequential criterion, it suffices to prove that any sequence of Hamiltonian homeomorphisms converging to the constant map identity with respect to the norm $\|\cdot\|_{S H L}$, converges to the constant map identity with respect to Oh's norm. The proof that we give here heavily relies the ideas that $\mathrm{Oh}$ [12] and Banyaga 2] used in the proof of the nondegeneracy of their norms. Let $\psi^{i}$ be a sequence of Hamiltonian homeomorphisms that converges to the identity with respect to the norm $\|\cdot\|_{S H L}$. For each $i$, and any $\epsilon>0$ there exists a strong symplectic isotopy $\psi_{\left(U^{i, \epsilon}, \mathcal{H}^{i, \epsilon}\right)}$ such that $\psi_{\left(u^{i, \epsilon}, \mathcal{H}^{i, \epsilon}\right)}^{1}=\psi^{i}$, and

$$
l_{\infty}\left(\psi_{\left(U^{i, \epsilon}, \mathcal{H}^{i, \epsilon}\right)}\right)<\left\|\psi^{i}\right\|_{S H L}+\epsilon .
$$

On the other hand, for a fixed $i$, there exists a sequence of symplectic isotopies $\phi_{\left(V_{i, j}, \mathcal{K}_{i, j}\right)}$ such that

$$
\psi_{\left(U^{i, \epsilon}, \mathcal{H}^{i, \epsilon}\right)}=\lim _{\mathrm{C}^{\mathcal{O}+\mathrm{L}^{\infty}}}\left(\phi\left(\mathrm{V}_{i, j}, \mathcal{K}_{i, j}\right)\right)
$$

In particular, one can find a sufficiently large integer $j_{0}$ such that $\phi_{\left(\mathrm{V}_{i, j_{0}}, \mathcal{K}_{i, j_{0}}\right)}$ is sufficiently close to $\psi_{\left(\mathrm{U}^{i, \epsilon}, \mathcal{H}^{i, \epsilon}\right)}$ with respect to the $\left(\mathrm{C}^{0}+\mathrm{L}^{\infty}\right)$-topology, and so that

$$
l_{\infty}\left(\psi_{\left(U^{i, \epsilon}, \mathcal{H}^{i, \epsilon}\right)}\right)>l_{\infty}\left(\phi\left(V_{i, j_{0}}, \mathcal{K}_{i, j_{0}}\right)\right)-\frac{\epsilon}{4} .
$$

Since $\left\|\psi^{i}\right\|_{\text {SHL }} \rightarrow 0, i \rightarrow \infty$, then the Hofer-like length of the isotopy $\phi_{\left(V_{i, j_{0}}, \mathcal{K}_{i, j_{0}}\right)}$ can be considered as being sufficiently small for $i$ sufficiently large. This implies that the flux of the path $\phi_{\left(V_{i, j_{0}}, \mathcal{K}_{i, j_{0}}\right)}$ can be considered as arbitrarily small for all $i$ sufficiently large. Hence, it follows from Banyaga 2] that, for all $i$ sufficiently large, the time-1 map of $\phi_{\left(V_{i, j_{0}}, \mathcal{K}_{i, j_{0}}\right)}$ is a Hamiltonian diffeomorphism. So, we can assume (without breaking the generality) that $\phi_{\left(\mathrm{V}_{i, j_{0},}, \mathcal{K}_{\left.i, j_{0}\right)}\right)}$ is Hamiltonian for $i \leq j_{0}$, and $i$ sufficiently large. Therefore, the above statements together with formula (5.18) imply that

$$
l_{\infty}\left(\psi_{\left(\mathrm{U}^{i, \epsilon}, \mathcal{H}^{i, \epsilon}\right)}\right)>\left\|\phi_{\left(\mathrm{V}_{i, j_{0}}, \mathcal{K}_{i, j_{0}}\right)}^{1}\right\|_{H L}-\frac{\epsilon}{4} .
$$

For $i \leq j_{0}$, and $i$ sufficiently large, since the diffeomorphism $\phi_{\left(\vee_{i, j_{0}}, \mathcal{K}_{i, j_{0}}\right)}^{1}$ is Hamiltonian, we derive from a result found in [7, 16] that there exists a positive finite constant D which does not depend on neither $i$, nor $j_{0}$, such that

$$
\frac{1}{\mathrm{D}}\left\|\phi_{\left(\mathrm{V}_{i, j_{0}}, \mathcal{K}_{i, j_{0}}\right)}^{1}\right\|_{H} \leq\left\|\phi_{\left(\mathrm{V}_{i, j_{0}}, \mathcal{K}_{i, j_{0}}\right)}^{1}\right\|_{S H L},
$$


where $\|\cdot\|_{H}$ represents the Hofer norm of Hamiltonian diffeomorphisms. This implies that

$$
\left\|\psi^{i}\right\|_{S H L}+\epsilon>\frac{1}{D}\left\|\phi_{\left(V_{i, j_{0}}, \mathcal{K}_{i, j_{0}}\right)}^{1}\right\|_{H}-\frac{\epsilon}{4},
$$

for $i \leq j_{0}$, and $i$ sufficiently large. At this level, we use the fact that Oh's norm restricted to the group of Hamiltonian diffeomorphisms is bounded from above by Hofer's norm to get

$$
\left\|\psi^{i}\right\|_{S H L}+\epsilon>\frac{1}{D}\left\|\phi_{\left(V_{i, j_{0}}, \mathcal{K}_{i, j_{0}}\right)}^{1}\right\|_{O h}-\frac{\epsilon}{4},
$$

for $i \leq j_{0}$, and $i$ sufficiently large. Passing to the limit in the latter estimate, yields

$$
\lim _{i \rightarrow \infty}\left\|\psi^{i}\right\|_{S H L}+\frac{5 \epsilon}{4} \geq \frac{1}{D} \lim _{j_{0} \geq i, i \rightarrow \infty}\left\|\phi_{\left(V_{i, j_{0}}, \mathcal{K}_{i, j_{0}}\right)}^{1}\right\|_{O h}=\frac{1}{\mathrm{D}} \lim _{i \rightarrow \infty}\left\|\psi^{i}\right\|_{O h},
$$

for all $\epsilon$. Finally, we have proved that for all positive real number $\delta$ (replacing $\epsilon$ by $\frac{4 \delta}{5 \mathrm{D}}$ ), we have

$$
\delta \geq \lim _{i \rightarrow \infty}\left\|\psi^{i}\right\|_{\text {Oh }}
$$

This completes the proof. $\square$

\subsection{Topological symplectic displacement energy}

In this Section, we extend the symplectic displacement energy to the world of strong symplectic homeomorphisms. This is motivated by the uniqueness result from [5] and the uniqueness of Banyaga's Hofer-like geometry [15].

Definition 5.6. The strong symplectic displacement energy $e_{S}^{0, \infty}(B)$ of a non empty compact subset $B \subset M$ is :

$$
e_{S}^{0, \infty}(B)=\inf \left\{\|h\|_{S H L} \mid h \in \operatorname{SSympeo}(M, \omega), h(B) \cap B=\emptyset\right\} .
$$

Lemma 5.7. For any non empty compact subset $\mathrm{B} \subset \mathrm{M}, \mathrm{e}_{\mathrm{S}}^{0, \infty}(\mathrm{B})$ is a strict positive number.

Proof. Let $\epsilon>0$, by definition of $e_{\mathrm{S}}^{0, \infty}(\mathrm{B})$, there exists a strong symplectic isotopy $\psi_{\left(F^{\epsilon}, \lambda^{\epsilon}\right)}$ such that $\psi_{\left(\mathrm{F}^{\epsilon}, \lambda \epsilon\right)}^{1}=\mathrm{h}$, and

$$
e_{S}^{0, \infty}(B)+\frac{\epsilon}{2}>l_{\infty}\left(\psi_{\left(F^{e}, \lambda^{e}\right)}\right) .
$$

On the other hand, there exists a sequence of symplectic isotopies $\left(\phi_{\left(F_{i}, \lambda_{i}\right)}\right)$ that converges to $\psi_{\left(F^{e}, \lambda^{e}\right)}$ with respect to the $\left(C^{0}+L^{\infty}\right)$-topology. So, one can choose integer $j_{0}$ large enough such that $\phi_{\left(\mathrm{F}_{i}, \lambda_{i}\right)}^{1}$ displaces $\mathrm{B}$, and

$$
l_{\infty}\left(\psi_{\left(F^{\epsilon}, \lambda^{\epsilon}\right)}\right)>l_{\infty}\left(\phi_{\left(F_{i}, \lambda_{i}\right)}\right)-\frac{\epsilon}{4} .
$$


for all $i>j_{0}$. It follows from the definition of symplectic displacement energy that

$$
\left\|\phi_{\left(\mathrm{F}_{i}, \lambda_{i}\right)}^{1}\right\|_{H L} \geq e_{S}(B)>0,
$$

for all $i>j_{0}$. Relations (5.25), (5.26) and (5.27) implies that

$$
e_{S}^{0, \infty}(B)+\frac{\epsilon}{2}+\frac{\epsilon}{4}>l_{\infty}\left(\phi_{\left(F_{i}, \lambda_{i}\right)}\right) \geq\left\|\phi_{\left(F_{i}, \lambda_{i}\right)}^{1}\right\|_{H L} \geq e_{S}(B)>0,
$$

for $i$ sufficiently large. Therefore,

$$
e_{S}^{0, \infty}(B)+\epsilon>e_{S}(B)>0,
$$

for all positive $\epsilon$. This completes the proof.

\section{Conjectures and Examples}

\subsection{Conjectures:}

Conjecture $(A)$ : For any $h \in \operatorname{SSympeo}(M, \omega)$, we have $\|h\|_{S H L}=\|h\|_{S H L}^{(1, \infty)}$.

Conjecture-(A) is supported by the uniqueness result of Hofer-like geometry from [15] or more generally in [16].

Conjecture (B): If $h \in \operatorname{SSympeo}(M, \omega)$, then the norm $\phi \mapsto\left\|h \circ \phi \circ h^{-1}\right\|_{S H L}$ is equivalent to the norm $\phi \mapsto\|\phi\|_{S H L}$.

Conjecture-(B) is supported by a result found in [4] (Theorem 7).

Conjecture (C): Let $\sigma$ be the canonical volume form on the unit circle $\mathbb{S}^{1}$ given by the orientation of the circle. If $\lambda$ is a strong symplectic isotopy generated by $(\mathrm{U}, \mathcal{H})$, then the Fathi's mass flow of $\lambda$ is exactly one of the following quantities:

$$
\pm \frac{1}{(n-1) !} \int_{M}\left(\left(\int_{0}^{1} \mathcal{H}_{t} d t\right) \wedge \omega^{n-1} \wedge f^{*}(\sigma)\right),
$$

for any mapping $f: M \rightarrow \mathbb{S}^{1} . \mathcal{}$

In particular, any continuous Hamiltonian flow has a trivial mass flow. Therefore, is any strong symplectic isotopy with trivial Fathi's mass flow homotopic (relatively to fixed endpoints) to a continuous Hamiltonian flow? 
Conjecture (D): If $\lambda$ is a strong symplectic isotopy generated by $(\mathrm{U}, \mathcal{H})$, then the mapping, $\lambda \mapsto\left(\int_{0}^{1}\left[\mathcal{H}_{t}\right] d t\right) \in \mathrm{H}^{1}(M, \mathbb{R})$, is a well defined group homomorphism which only depend on the homotopic class of $\lambda$ relatively to fixed ends, where [,] stands for the de Rham cohomology class.

\subsection{Examples}

\subsubsection{Example}

A harmonic 1-parameter group is an isotopy $\beta=\left\{\beta_{\mathrm{t}}\right\}$ generated by the vector field $X$ defined by $\mathfrak{\imath}(\mathrm{X}) \boldsymbol{\omega}=\mathcal{K}$, where $\mathcal{K}$ is a harmonic $1-$ form, and let $\phi$ be a non-smooth Hamiltonian homeomorphism [13]. By definition of $\phi$, there exists a sequence of Hamiltonian isotopies $\Phi_{j}$ which is Cauchy in $\left(\mathrm{C}^{0}+\mathrm{L}^{\infty}\right)$, and $\phi_{j}:=\Phi_{j}(1) \rightarrow \phi$, uniformly. The isotopy $\Psi_{j}: t \mapsto \phi_{j}^{-1} \circ \beta_{t} \circ \phi_{j}$, has time-1 map $\phi_{j}^{-1} \circ \beta_{1} \circ \phi_{j}$, and it is generated by $\left(\int_{0}^{1} \mathcal{K}\left(\dot{\Phi}_{j}(s)\right) \circ \Phi_{j}(s) d s, \mathcal{K}\right)$. In fact, the smooth function $x \mapsto \int_{0}^{1} \mathcal{K}\left(\dot{\Phi}_{j}(s)\right) \circ \Phi_{j}(s) \mathrm{ds}(x)$, does not depend on the choice of any isotopy with time-1 map $\phi_{j}:=\Phi_{j}(1)$ [5, 16, 18]. As it can be checked, the sequence of generators $\left(\int_{0}^{1} \mathcal{K}\left(\dot{\Phi}_{j}(s)\right) \circ \Phi_{j}(s) d s, \mathcal{K}\right)$ is Cauchy in $\mathrm{D}^{\infty}$ if and only if the sequence of functions $x \mapsto\left(\int_{0}^{1} \mathcal{K}\left(\dot{\Phi}_{j}(s)\right) \circ \Phi_{j}(s) d s\right)(x)$ is Cauchy in the $L^{\infty}-$ Hofer norm. But, Lemma 3.9 from [15, 16] shows that the sequence of functions $x \mapsto\left(\int_{0}^{1} \mathcal{K}\left(\dot{\Phi}_{j}(s)\right) \circ \Phi_{j}(s) d s\right)(x)$, is Cauchy in the $\mathrm{L}^{\infty}$-Hofer norm provided the sequence $\Phi_{j}$ is Cauchy in the metric $\overline{\mathrm{d}}$; which is the case. Thus, the latter converges in the complete metric space $\mathcal{N}^{O}([0,1] \times M, \mathbb{R})$ to a time-independent continuous function that we denote $F_{0}$. The strong symplectic isotopy $\Phi: t \mapsto \phi \circ \beta_{\mathrm{t}} \circ \phi^{-1}$, is generated by $\left(F_{0}, \mathcal{K}\right)$, and its time-1 map $x \mapsto\left(\phi \circ \beta_{1} \circ \phi^{-1}\right)(x)$ is not necessary $C^{1}$, but continuous. Hence, we have constructed separately an example of strong symplectic isotopy which is a 1 -parameter subgroup and whose generator is time independent. The Hofer-like length of $\Phi$ are given by

$$
l_{\infty}(\Phi)=\operatorname{osc}\left(F_{0}\right)+|\mathcal{K}|=l_{(1, \infty)}(\Phi)
$$

and we also have,

$$
\bar{e}_{0}\left(\phi \circ \beta_{1} \circ \phi^{-1}\right) \leq \bar{e}\left(\phi \circ \beta_{1} \circ \phi^{-1}\right) \leq \operatorname{osc}\left(F_{0}\right)+|\mathcal{K}|<\infty .
$$

$\triangle$

\subsubsection{Example}

Consider the torus $\mathbb{T}^{2 l}$ with coordinates $\left(\theta_{1}, \ldots, \theta_{2 l}\right)$ and equip it with the flat Riemannian metric go. Note that all the 1 -forms $d \theta_{i}, i=1, \ldots, 2 l$ are harmonic. Take the 1 -forms $d \theta_{i}$ for $i=1, \ldots, 2 l$ as basis for the space of harmonic 1 -forms and consider the symplectic form $\omega=\sum_{i=1}^{l} d \theta_{i} \wedge d \theta_{i+l}$. Given $v=\left(a_{1}, \ldots, a_{l}, b_{1}, \ldots, b_{l}\right) \in \mathbb{R}^{2 l}$, the translation $x \mapsto x+v$ on $\mathbb{R}^{2 l}$ induces a rotation $R_{v}$ on $\mathbb{T}^{2 l}$, which is a symplectic diffeomorphism. Therefore, the smooth mapping $\left\{R_{v}^{t}\right\}: t \mapsto R_{t v}$ defines a symplectic isotopy generated by $(0, \mathcal{H})$ with $\mathcal{H}=\sum_{i=1}^{l}\left(a_{i} d \theta_{i+l}-b_{i} d \theta_{i}\right)$. Now, consider the 
torus $\mathbb{T}^{2}$ as the square: $\square:=\{(p, q) \mid 0 \leq p \leq 1,0 \leq q \leq 1\} \subset \mathbb{R}^{2}$, with opposite sides identified. Then, the action of the unit circle $\mathbb{S}^{1}$ on $\mathbb{T}^{2}$ :

$$
\rho: \mathbb{S}^{1} \times \mathbb{T}^{2} \rightarrow \mathbb{T}^{2},\left(\alpha,\left(\theta_{1}, \theta_{2}\right)\right) \mapsto\left(\theta_{1}+\alpha, \theta_{2}+\alpha\right),
$$

induces a non-Hamiltonian diffeomorphism $\rho_{\alpha}: \mathbb{T}^{2} \rightarrow \mathbb{T}^{2}$, because the latter has no fixed point for $\alpha$ small and non-trivial. Assume this done. Let $\mathbb{D}^{2} \subset \mathbb{R}^{2}$ be the 2 -disk of radius $\left.\tau \in\right] 0,1 / 8[$ centered at $A=(a, 0)$ with $7 / 8 \leq a<1$, and let $\Lambda^{2}(\tau)$ be the corresponding subset in $\mathbb{T}^{2}$. For any $v<1 / 4$, consider the nonempty open subset $\mathrm{B}(v)=\{(x, y) \mid 0<x<v\} \subset \mathbb{R}^{2}$, and let $\mathrm{C}(v)$ be the corresponding open subset in $\mathbb{T}^{2}$.

Let $\phi$ be a non-smooth Hamiltonian homeomorphism of $\mathbb{T}^{2}$ supported in $\Lambda^{2}(\tau)$, and assume that $\rho_{\alpha}$ is induced by a horizontal vector $v=(b, 0) \in \mathbb{R}^{2}$, with $v<b \leq 1 / 2$. Then, the map $x \mapsto\left(\phi^{-1} \circ \rho_{\alpha} \circ \phi\right)(x)$ displaces completely the nonempty open subset $C(v)$. It follows from the proof of Lemma 5.7 that, $0<e_{S}(C(v)) \leq e_{S}^{0, \infty}(C(v)) \leq l_{\infty}\left(\left\{\phi^{-1} \circ \rho_{(\alpha \mathrm{t})} \circ \phi\right\}_{t \in[0,1]}\right)<\infty$.

\section{Acknowledgments:}

The first author thanks the African Academy of Sciences, the African Institute for Mathematical Sciences of South Africa, and the Chair of Mathematics of the African Institute for Mathematical Sciences of Senegal, for the financial support of his researches.

\section{References}

[1] A. Banyaga, : Sur la structure de difféomorphismes qui préservent une forme symplectique, comment. Math. Helv. 53 (1978), $174-2227$.

[2] A. Banyaga, : A Hofer-like metric on the group of symplectic diffeomorphisms, Contempt. Math. Amer. Math. Soc. RI. 512 (2010), 1 - 24.

[3] A. Banyaga,: On the group of strong symplectic homeomorphisms, C. R. Math. Acad. Sci. Paris 346 (2008), no. $15-16,867872$.

[4] A. Banyaga, E. Hurtubise, and P. Spaeth, :On the symplectic displacement energy, To appear in Journal of Symplectic Geometry (2015).

[5] A. Banyaga and S. Tchuiaga, :The group of strong symplectic homeomorphisms in $\mathrm{L}^{\infty}$-metric, Adv. Geom. 14 (2014), no. 3, 523539.

[6] A. Banyaga and S. Tchuiaga, : Uniqueness of generators of strong symplectic isotopies, Afr. J. Pure Appl. Math. 1, no 1, (2016), 7 - 23.

[7] G. Bus and R. Leclercq, : Pseudo-distance on symplectomorphisms groups and application to the flux theory, Math. Z 272 (2012) 1001 - 1022. 
[8] L. Buhovsky and S. Seyfaddini, Uniqueness of generating Hamiltonian for continuous Hamiltonian flows, J. Symp. Geom. 11 no. 1, (2013) $37-52$.

[9] Y. Eliashberg, : A theorem on the structure of wave fronts and its application in symplectic topology, Funct. Anal. and Its Applications 21 (1987) 227 - 232.

[10] M. Gromov, : Pseudoholomorphic cuves in symplectic manifold, Inent. Math. 82 (1985) $307-347$.

[11] H. Hofer and E. Zehnder, : Symplectic invariants and hamiltonian dynamicss, Birkhauser Advanced Texts, Birkhauser Verlag (1994).

[12] Y-G. Oh, : The group of Hamiltonian homeomorphisms and continuous Hamiltonian flows, submitted to the proceedings of the 2007 AMS-IMS-SIAM Summer Research Conference, Snowbird, Utah (2007).

[13] Y-G. Oh and S. Müller, : The group of Hamiltonian homeomorphisms and $\mathrm{C}^{0}$-symplectic topology, J. Symp. Geometry 5 (2007) $167-225$.

[14] S. Tchuiaga, : Some Structures of the Group of Strong Symplectic Homeomorphisms, G.J.A.R.C.M. Geometry, 2, Issue 1 (2013) 36-49.

[15] S. Tchuiaga, : On symplectic dynamics, Afri. D. J. Math. 20, Issue 2, (2017) 69 - 94.

[16] S. Tchuiaga, : On symplectic dynamics, To appear in DGA (2017).

[17] S. Tchuiaga, : Hofer-like Geometry and Flux theory, Preprint (2016).

[18] S. Tchuiaga, : An enlargement of some symplectic objects, Arxiv: 1410.7891v6 [math. SG], 04 January (2016).

[19] C, Viterbo, : On the uniqueness of generating Hamiltonian for continuous limits of Hamiltonian flows, Internat. Math. Res. Notices, vol (2006), Article ID 34028, 9 pages. Erratum, ibid Vol (2006) article ID 38784, 4 pages.

[20] F. Warner, Foundation of differentiable manifolds and Lie groups, Graduate Texts in Mathematics, 94, Springer-Verlag, New York, (1983). 